\title{
Syphilitic aortitis: The bigger picture
}

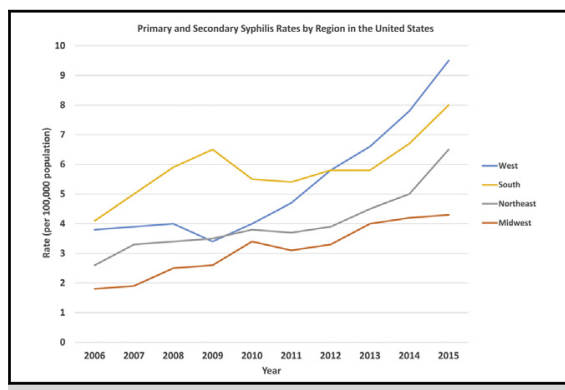

The incidence of syphilis is increasing across all regions of the United States.

\section{Central Message}

Syphilitic aortitis commonly leads to ascending aortic aneurysms. Complications such as rupture or fistula formation are common. Successful surgery and postoperative antibiotics can be curative.

See Article page e23. case of this resurgent disease can have profound epidemiologic impact.

In 2015, the Centers for Disease Control and Prevention reported 74,702 new cases of syphilis in the United States. This was nearly double the reported incidence of human immunodeficiency virus infection during the same year. The

overall number of cases has increased during the last 6 years in all regions of the United States and is not limited to urban populations (Figure 1). ${ }^{2}$ The infection arises from exposure to the bacterium Treponema pallidum, and untreated it can

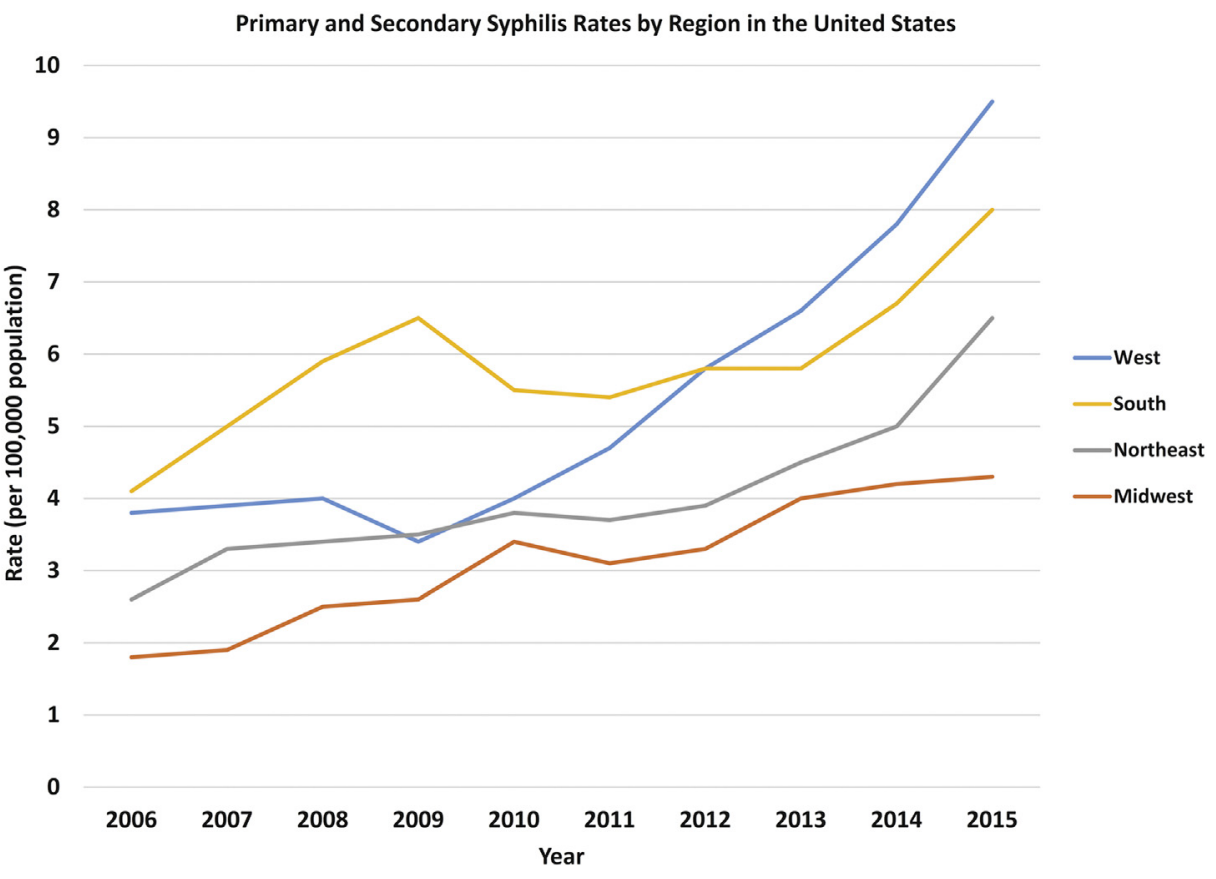

FIGURE 1. Centers for Disease Control and Prevention graph of primary and secondary syphilis rates of reported cases by region in the United States, 2006 to 2015 . $^{2}$ 
lead to serious health sequelae, including neurologic, ocular, cardiovascular, and congenital syphilis. Tertiary syphilis, as seen in this case report, can appear 10 to 30 years after the infection was acquired. The diagnosis can be made by nontreponemal testing (Venereal Disease Research Laboratory rapid plasma reagent), but this is fairly nonspecific, and treponemal antibody testing (as in this case) is often required to confirm the diagnosis. ${ }^{3}$ Syphilitic aneurysms have a 2-year mortality greater than $80 \%$ when left untreated. Most of the deaths are attributable to aortic rupture or fistulization into adjacent structures, because the obliterative endarteritis of the vasa vasorum infection leads to an intense inflammatory reaction that creates these complicated situations. $^{4}$

In one of the larger reviews on syphilitic aneurysms, Roberts and colleagues ${ }^{5}$ found that the mean age at presentation was 66 years, but the range of ages presenting with aneurysm in their series was 33 to 84 years of age. There was no gender bias, but $78 \%$ of those specimens examined came from Caucasians. There was a fusiform aneurysm of the ascending aorta in all 23 cases, 52\% had arch involvement, and $43 \%$ had descending aortic disease. The abdominal aorta was not involved. Fewer than one-quarter had a saccular component on top of the fusiform aneurysm, and very few had positive nontreponemal serologic results at the time of presentation. All cases of syphilitic aortic involvement were confirmed after surgery by either antibody testing or pathology.

The treatment for syphilis is quite effective and dependent on the stage of disease at presentation. As Isbir and colleagues ${ }^{1}$ note in their case report, patients with a diagnosis of tertiary syphilitic aortitis will require 21 days of therapy with benzathine penicillin after surgery. Lifelong surveillance of the remainder of the aorta is required because of the high incidence of downstream aortic involvement. Although we are surgeons, the diagnosis begins with us. Increased awareness can make a significant positive contribution to the successful treatment of this concerning and growing epidemiologic scourge.

\section{References}

1. İsbir S, Hamidov A, Seven IE, Ak K. Massive hemoptysis related to contained rupture of syphilitic aortic aneurysm into the pulmonary parenchyma. J Thorac Cardiovasc Surg. 2017;154:e23-5.

2. Centers for Disease Control and Prevention. Figure 33. Primary and secondary syphilis—rates of reported cases by region, United States, 2006-2015. Available at: www.cdc.gov/std/stats15/figures/33.htm.

3. Ghanem KG. Management of adult syphilis: key questions to inform the 2015 Centers for Disease Control and Prevention sexually transmitted diseases treatment guidelines. Clin Infect Dis. 2015;61:S818-36.

4. Maleszewski JJ. Inflammatory ascending aortic disease: perspectives from pathology. J Thorac Cardiovasc Surg. 2015;149(2 Suppl):S176-83.

5. Roberts WC, Barbin CM, Weissenborn MR, Ko JM, Henry AC. Syphilis as a cause of thoracic aortic aneurysm. Am J Cardiol. 2015;116:1298-303. 